les valeurs les plus diverses : 4 pour la Somme, 3 io pour le Rhône à son embouchure dans le lac de Genève, 24 pour le lac de Genève, à Genève, I 400 pour la Loire, à Roanne, 1700 pour l'Ardèche à son confluent dans le Rhône, etc. Les torrents sont caractérisés par les grandes valeurs du coefficient de crue.

\section{5o Conséquences.}

De cet exposé, il résulte qu'en pratique les éléments : débit, hauteur de chute, puissance, doivent être rapportés à des unités spécifiques bien définies pour pouvoir, en langage ordinaire, présenter un sens concrèt .

Il en résulte aussi qu'en adjoignant le coefficient de crue aux mesures de la chute faites dans les unités particulières on définit les circonstances extrêmes de l'existence de cette chute, de façon à en complèter le signalement de la manière la plus pratique.

Il semble, dès lors, possible de comparer a priori, les différentes propriétés des chutes exprimées en mots du langage usuel, qu'on fait ainsi peser dans le discours d'un poids précis et défini. L'usage et la raison voient ainsi leurs exigences conciliés.

C'est là un résultat dont l'importance pratique nous semble réelle, par la clarté qu'il permet de mettre désormais dans les études et par la manière dont il peut rendre fructueux les rapprochements entre les propriétés des différentes chutes.

$\mathrm{Si}$, par exemple, un grand lac équatorial se déverse par une arète de 3 kilomètres de long et débite $3000 \mathrm{~m}^{3}$ par seconde avec $50 \mathrm{~m}$. de chute, nous dirons que nous avons là une basse chute, à petite puissance spécifique, à très gros débit spécifique, donnant la puissance absolue considérable de 15 ooo ooo poncelets.

$\mathrm{Si}$, de plus, son coefficient de crue est de 24 , comme il semble être pour le Léman, nous voyons que lorsque les eaux deviennent abondantes, sa puissance peut atteindre une valeur très considérable ( $\mathrm{I}$ )

En regard de ce lac, supposons une cascade de $800 \mathrm{~m}$. de chute, de o m. 5o de large, avec un débit de 100 litres. Son débit spécifique serait petit $\left(\frac{100}{0,5}=200\right.$ litres $)$ sa hauteur, donc sa puissance spécifique, très grande ( 8 poncelets). Sa puissance absolue, en se reportant au tableau I, sera considérée comme grande, mais sans excès (2).

(1) La rapidité des crues et des décrues, très importante en pratique, est étrangère à notre thèse, encore qu'elle s'y rattache ici. Elle fera l'objet d'autres études. Elle est. comme chacun sait, directement intluencée par la superficie du lac, par sa forme plus ou moins épanouie, par la nature du sol de ses rives, leur état de dénudation ou de gazonnement et de boisement, les vents régnants, la fréquence et l'abondance des pluies, etc., etc. Plus le cours d'eau est resserré, plus toutes choses égales d'ailleurs, crues et décrues sont brusques.

(2) A supposer qu'une verticale de $800 \mathrm{~m}$. existe quelque part dans la nature, ce qui doit être rare, il est bien présumable qu'une masse d'eau de faible importance comme celle-ci, arriverait presque entièrement à l'état pulvérulent en bas de la chute. En effet, les vents d'ouragan soulèvent des embruns en rasant la surface de la mer, leur vitesse n'est que de $45 \mathrm{~m}$. ̀̀ $50 \mathrm{~m}$. par seconde. Cette valeur de la vitesse est acquise par une chute de $100 \mathrm{~m}$. Elle atteindrait $125 \mathrm{~m}$.
Mais si cette chute provient d'un torrent dont le coefficient de crue soit r 700 , comme celui de l'Ardèche, on est tout de suite renseigné sur la violence extrême des effets qu'elle peut produire.

Cette manière d'évaluer les diverses propriété de la chute, met donc bien en évidence la valeur relative de chacun des facteurs de la puissance, et permet de préciser le langage usuel par lequel on les caractérise. C'est le résultat que nous avions en vue en abordant cette étude.

Commandant Audraraxi, Ancien élève de l'Ecole Polytechnique, Ingénieur.

\section{Sup la Pabrication synthétique de l'Aleool}

\section{en partant du carbure de calcium}

L'utilisation industrielle de l'alcool à l'éclairage, au chauffage et principalement à la production de la force motrice,entre dans une voie qui semble devoir être féconde en résultats pratiques. Et pour peu que,cette utilisation se généralise, les producteurs actuels d'alcool n'auront pas les moyens de faire face aux exigences de la consommation. On poussera l'agriculture à satisfaire aux besoins de cette nouvelle industrie, mais on peut se demander si notre sol sera assez riche pour alimenter toutes les fabriques d'alcool qu'un avenir, peut-être prochain, nécessitera.

Il nous paraît donc d'actualité de rappeler ici un procédé de fabrication artificielle de l'alcool qui mettrait en œuvre d'autres ressources dont notre pays est également si riche et qui même paraissent inépuisables: nous voulons parler des forces motrices bydrauliques. On peut en effet faire de l'alcool de toutes pièces, en partant du carbure de calcium; opérer sa synthèse en empruntant, par voie chimique, au charbon et à l'eau tous ses éléments constitutifs. Ce procédé auquel on n'a pu songer que depuis la fabrication industrielle du carbure de calcium, intéresse donc grandement l'industrie, appelée à vivre de la Houille blanche.

Le principe de la préparation synthétique de l'alcool est le suivant :

L'acétylène $\mathrm{C}^{2} \mathrm{H}^{2}$ est susceptible de s'hydrogéner en se transformant en éthylène $\mathrm{C}^{2} \mathrm{H}^{4}$.

D'autre part, l'éthylène, en se combinant aux éléments de l'eau, donne l'alcool éthylique $\mathrm{C}^{2} \mathrm{H}^{6} \mathrm{O}$.

Il y aura donc, pour opérer cette synthèse, deux réactions à produire, représentées par les équations suivantes:

$I^{0}$

$2^{\circ}$

$$
\begin{gathered}
\mathrm{C}^{2} \mathrm{H}^{2}+2 \mathrm{H}=\mathrm{C}^{2} \mathrm{H}^{4} \\
\mathrm{C}^{2} \mathrm{H}^{4}+\mathrm{H}^{2} \mathrm{O}=\mathrm{C}^{2} \mathrm{H}^{0} \mathrm{O}
\end{gathered}
$$

pour $800 \mathrm{~m}$. On voit, sans insister, quelle facilité l'air a de pénétrer une masse d'eau quelque peu gracile. Nous nous sommes donc placés dans un cas que la nature n'offrira pas spontanément, mais rien n'empêche de racheter $800 \mathrm{~m}$. de'différence de niveau par une conduite forcée, et alors les conditions de norre exemple deviennent réelles. 
Mais, si l'acétylène peut se combiner directement à l'hydrogène pour donner naissance à de l'éthylène, l'éthylène, lui, ne se combinera pas directement aux éléments de l'eau pour former l'alcool. Il faudra, pour réaliser cette deuxième phase de l'opération, avoir rẹcours à un intermédiaire qui sera l'acide éthylsufurique, appelé encore acide sulfovinique; ce corps n'est autre chose que de l'acide sulfurique $\mathrm{SO}^{4} \mathrm{H}^{2}$ dans lequel un atome d'hydrogène $\mathrm{H}$ est remplacée par le groupe éthyl $\mathrm{C}^{2} \mathrm{H}^{5}$, et qui a,par conséquent, pour formule $\mathrm{SO}^{4} \mathrm{H} \mathrm{C}^{2} \mathrm{H}^{5}$. Or, l'acide sulfovinique, en présence de l'eau, et à l'ébulition, se dédouble en acide sulfurique et en alcool, d'après l'équation:

$$
\mathrm{SO}^{4} \mathrm{H} \mathrm{C}^{2} \mathrm{H}^{5}+\mathrm{H}^{2} \mathrm{O}=\mathrm{SO}^{4} \mathrm{H}^{2}+\mathrm{C}^{2} \mathrm{H}^{6} \mathrm{O}
$$

Quant à l'acide sulfovinique, c'esta-à-dire l'intermédiaire en question, on le produira avec l'éthylène obtenu au moyen de l'acétylène. En effet, l'éthylène, en présence de l'acide sulfurique se dissout dans cet acide, sous conditions d'élever la température de ce dernier corps et d'agiter le mélange d'éthylène et d'acide. Il se forme alors de l'acide sulfovinique et la réaction s'exprime de la manière suivante:

$$
\mathrm{C}^{2} \mathrm{H}^{4}+\mathrm{SO}^{4} \mathrm{H}^{2}=\mathrm{SO}^{4} \mathrm{H} \mathrm{C}^{2} \mathrm{H}^{5}
$$

Ces principes théoriques étant exposés, voici comment on peut réaliser pratiquement cette synthèse. On se servira de l'appareil représenté par la figure ci-contre, permettant de préparer, en laboratoire, l'alcool artificiel.

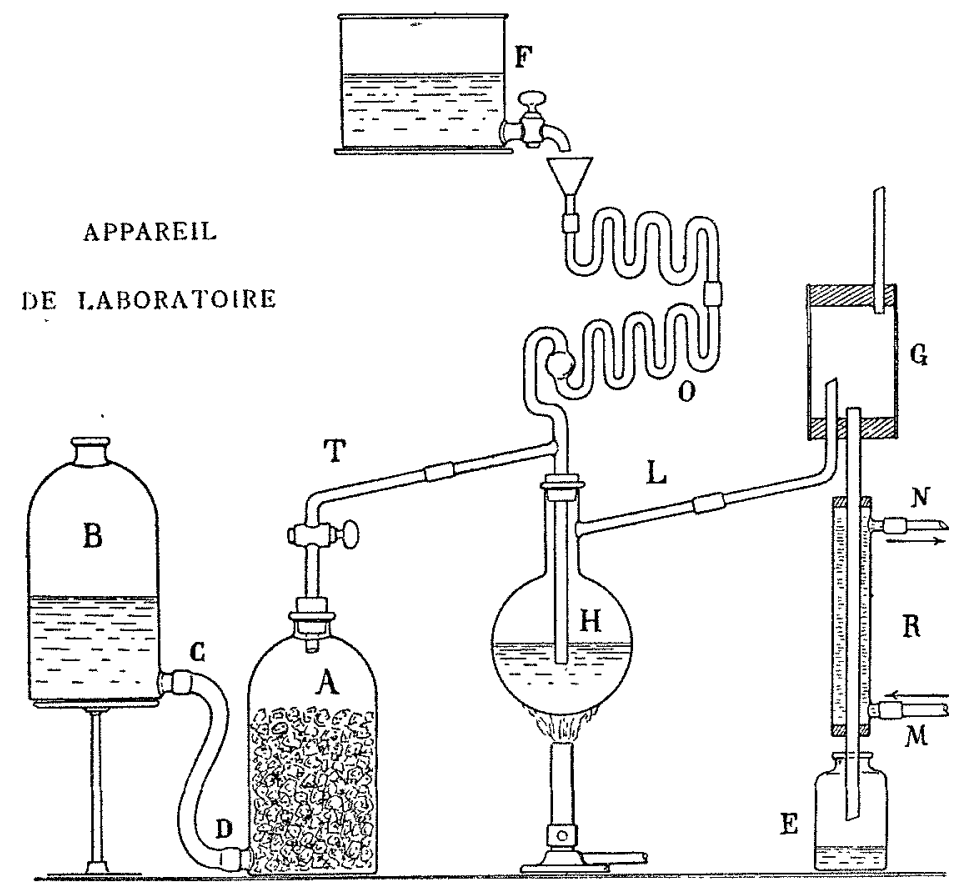

Préparation de l'alcool en partant du carbure de calcium.

Les deux flacons, $\mathrm{A}$ et $\mathrm{B}$ constituent un appareil Sainte-Claire-Deville, si connu qu'il est inutile de le décrire.

Le flacon A contient un mélange de carbure de calcium et de zinc dans la proportion de 2 kil. de carbure pour 2 kil. $1 / 2$ de zinc.

Dans le flacon $B$ on met un mélange d'eau et d'acide sulfurique (5 litres d'eau et 3 kil. 200 d'acide sulfurique). Par suite de la différence de niveau des deux flacons l'eau aciludée, passant par le tube $C D$, arrive en contact avec le carbure et le zinc, et attaque ces deux corps; il se produit de l'hydrngène et de l'acétylène. Les deux gaz se combinent pour former de l'éthylène qui se dégage par le tube T. La première phase de l'opération (transformation de l'acétylène en éthylène) est donc à ce moment réalisée.

Dans le récipient $F$ on a placé de l'acide sulfurique chaud que l'on fait alors couler par petites quantités en réglant le robinet. L'acide sulfürique, passant dans l'appareil $\mathrm{O}$, rencontre l'éthylène qui se dégage par le tube T. Il ya ainsi formation d'acide sulfovinique qui tombe graduellement dans ballon $\mathrm{H}$ contenant une certaine quantité d'eau portée à l'ébullition.

C'est dans ce ballon que se produit la deuxième phase de l'opération (décomposition de l'acide sulfovinique en acide sulfurique et alcool).

L'alcool dégagé en vapeur, se rend, par le tube $L$, dans un premier réfrigérent $G$, puis un second $R$ à courant d'eau ascendant $\mathrm{M} N$ et finalement distille dans le flacon $\mathrm{E}$.

On peut, au lieu de produire l'hydrogène par la réaction de l'acide sulfurique sur le zinc, employer l'hydrogène obtenu par électrolyse de l'eau et qui remplit parfaitement les conditions voulues.

L'alcool obtenu par ce procédé est pur et ne contient pas d'aldéhydes.

11 serait assurément prématuré de chercher à établir des rendements. Ces expériences réussissent en laboratoire. Donneraient-elle des résultats, reproduites à une échelle industrielle? Sans doute, mais nous ne pouvons dire lesquels et nousignorons si lachose a été tentée. En tous cas elle mérite de l'être. Il est en effet, certain que, du jour oủ l'utilisation industrielle de l'alcool sera un fait accompli, sa consommation deviendra énorme et alors sa fabrication artificielle pourrait être l'une des . branches les plus impor. tantes de l'industrie hydro-électrique. Et si, même, il nous était permis d'aller, par la pensée, dans l'avenir, nous dirions que les pays étrangers seront tributaires de la France pour l'alcool, comme elle l'est elle-même, aujourd'hui, des pays riches en pétrole.

Le prix de revient de l'alcool de synthèse dépendra principalement de celui du carbure de calcium qui en est le.point de départ. Aujourd'hui on abandonne sa fabrication parce que les débouchés de ce produit sont insuffisants et il arrive - ce qui a toujours lieu en semblable circonstance - que. devant les mécompres de la partie commerciale, la partie technique abandonne toutes recherches et tous essais de perfectionnement. C'est, à cette cause que l'on peut, partiellement du moins, attribuer l'imperfection relative des fours à carbure et le prix de revient élevé du produit. Mais qu'un nouveau débouché soit ouvert et l'on verra immédiatement les chercheurs se remettre à l'œuvre et trouver les appareils économiques qui font défaut pour le moment.

$\mathrm{Si}$, par ce court exposé de faits et d'idées nous avions réussi à déterminer quelques-uns de ces chercheurs à travailler dans cette voie nous estimerions avoir atteint le but que nous nous sommes proposé en écrivant ces lignes.

\section{D'Hector de Rochefontainf,}

Chimiste,

Expert près les Tribunaux. 